\title{
Groups: Toward a Theory of Plural Embodiment
}

\section{Gabriel Uzquiano}

\section{$1 \quad$ Introduction}

Groups are ubiquitous in our lives. Committees link several individuals united by a common purpose, a principle of organization, and a set of rules and procedures. Like committees, courts and orchestras are highly structured and appear to support a shared intentionality and agency. Other groups seem much less cohesive: neither queues nor multitudes appear to demand much of their individual members. They must exemplify a certain spatial arrangement but they do not seem to require a shared intentionality or agency from their members. And there are of course many cases in between. The purpose of this paper is to address the metaphysical question of what exactly is a group. An answer to this question ought to accommodate some of the differences mentioned above, and it should satisfy at least four additional constraints.

We should first do justice to the observation that groups may have their members temporarily and contingently. We join a committee for a period of time, but fortunately, we are eventually allowed to leave. Nor is it necessary that we join the committee; it would have remained the same committee if someone else had joined instead.

Second, we should make allowance for coincidence. Two groups may share exactly the same members at a given time. Two different committees may coincide even if they are governed by a different purpose, a different principle of organization, and a different set of rules and procedures. The search committee and the graduate committee may share exactly the same members throughout the week. The very individuals who meet on Tuesday as the members of the search committee may meet on Thursday as the members of the graduate committee. This is not to say of course that the graduate committee has met on Tuesday or that the search committee has met on Thursday. Nor is it better to say that the search committee does not exist when the graduate committee is in session - or vice versa.

We should, in the third place, explain what is for an individual to be a member of a given group at a time. It would be better of course if the account could illuminate the distinction between highly structured groups with their apparent shared intentionality and agency like committees and courts and apparently less cohesive groups such as queues and multitudes.

Finally, we should, if we can, explain what is for a group to be part of another. This, unlike group membership, appears to be an 
atemporal relation. The senior search committee is part of the search committee throughout different changes in membership. Given the allowance for coincidence, two groups may coincide at a given time even if only one of them is part of a larger group: the senior search committee is part of the search committee of which the junior search committee is another part, but the mere fact that the search committee coincides with the graduate committee at a given time is not enough of course to make the graduate committee part of the search committee.

There is by now a large literature on the metaphysics of groups. There is, first, the view that a group is nothing over and above their members. Groups, on this view, are pluralities of individuals. ${ }^{1}$ One challenge for this approach is to accommodate the first constraint: if an individual is one of some individuals, then it is one of them at all times and worlds at which they exist. And there is the fact that pluralities are completely individuated by their members: two pluralities differ only if they have different members. So, it is difficult to accommodate the fact that different groups can share exactly the same members. ${ }^{2}$

A more sophisticated approach has been defended by [Simons, $1987,4.4$. An orchestra is given by some musicians as they satisfy certain constitution conditions, which require to meet regularly to play a certain array of instruments, and so on. This allows him to accommodate that a musician may only temporarily and contingently be a member of an orchestra, but questions remain: what makes some musicians at one time be one and the same orchestra as some other musicians at a later time? And how are we to explain group coincidence? If some individuals satisfy the constitution conditions associated with one and the other, then it is still not entirely clear why we have two groups and not one.

Similar difficulties threaten the identification of a group with the set of its members. There are, however, other alternatives in the vicinity: [Effingham, 2010] has recently advocated the identification of groups with sets - not of their members - of ordered pairs of worlds and ordered pairs of times and sets of members. The account is tailor-made to explain how groups allow for variation in their members across times and worlds, and it makes allowance for two different groups to share exactly the same members at a given time - and even at all times at a given world. But it does less well with the third constraint: to be a member of a group at a given time at a world is to appear in the second component of a certain ordered pair in one of the ordered pairs in the set indexed by the relevant world. The difference between a committee and a queue amounts to a difference in extension, which is not very illuminating.

\footnotetext{
${ }^{1}$ It is often convenient to speak in the singular of a plurality of objects to refer in the plural to some objects. And we likewise speak of an individual object as a member of a plurality to mean that the individual object is one of them. Readers could in principle eliminate all these uses in favor of plural quantification and talk of the relation an object bears to some objects of which it is one.

${ }^{2}$ These points are discussed in great detail in [Ritchie, 2013].
} 
Next comes the identification of groups with fusions of their members. One advantage of this view is that it construes groups as concrete material objects of a piece with their members. And indeed, this may have been the predominant view of groups for some time. ${ }^{3}$ The identification of groups with fusions of their members eventually fell out favor partly as a result of the work of David-Hillel Ruben in [Ruben, 1983] and [Ruben, 1985]. But the approach is far from dead and has recently been revived by [Hawley, 2017]. This general line of attack is nevertheless very sensitive to the details of the general conception of mereological composition in the background.

On a familiar model of composition, a fusion is partially identical to its parts and moreover, has them eternally and necessarily if at all. This makes the thesis no less problematic than the identification of groups with their members. Moreover, this is a model of composition on which no two different fusions share exactly the same parts, which makes it difficult to accommodate coincidence. Some philosophers appeal to temporal parts in order to respond to some of these problems: [Copp, 1984] and [Sider, 2001, pp. 151-2] have defended the identification of groups with fusions of temporal parts. But it is less clear that they are able to satisfy some of the other constraints we set for ourselves: one challenge, for example, is to illuminate the distinction between a committee and a queue, which for them, is merely a different in parts.

We do better if we set the proposal against the background of a hylomorphic conception of composition, one on which at a given time, a queue, for example, consists of a composite material object whose immediate parts are individual members as they are unified by a certain complex spatial relation and a common purpose. We will in fact describe such a proposal in detail as a stepping stone towards the proposal to be defended in this paper.

This brief summary is far from exhaustive. We would be remiss if we did not mention at least two more options. Some philosophers have appealed to the concept of constitution: groups are not sets of individual members, but they are constituted by them. This relation is supposed to be akin to material constitution, which is the relation that a statue, for example, bears to the portion of clay of which it is made. Much like the statue is less closely tied to its parts than the clay is, [Uzquiano, 2004], that groups are less closely tied to their members than sets are to their elements. Different accounts of the relation a group bears to the set of its members are in line with different accounts of material constitution: [Epstein, 2015] has recently advocated an account of group constitution in terms of how facts to do with groups are grounded on facts to do with their individual members. There is a price to pay in each case: groups are complex
3 [Quinton, 1975] and [Macdonald and Pettit, 1981] are cases in point. 
objects of an unfamiliar sort, since they cannot quite be identified with material objects or sets thereof.

More recently, [Ritchie, 2013] has taken groups to be concrete realizations of abstract structures. A committee exemplifies an abstract structure, which includes nodes for the occupants of different roles in the committee and stipulates that certain relations obtain between them. There is a question as to whether this concrete realizations of abstract structures are more familiar objects than the complex objects posited by constitution theorists. And the view appears to be tailor-made to deal with committees and courts but less well suited to accommodate queues and multitudes.

While some accounts do better than others with the constraints outlined at the outset, most of them fail to do justice to the judgment that at a given time, a queue is nothing over and above some individuals as they stand in line for a certain purpose. Maybe there is a reason for this. One could be forgiven to think the task is unattainable: no answer to the question of what is a group can satisfy the constraints we lay out at the outset and nevertheless do justice to the judgment that at a given time, a group is nothing over and above some individuals as they exemplify a certain complex condition. One of the primary purposes of this paper is to suggest otherwise.

This is of course a tall order. So, we proceed in two steps. We first articulate a hylomorphic conception of groups that promises to satisfy the initial four constraints but does less well with the judgment in question. This first pass cannot be the end of the story for another reason: we will eventually argue that given reasonable assumptions, it leaves us on the brink of inconsistency. The second step is to offer a structurally similar account designed to reap all the benefits of the initial account and nevertheless accommodate the judgement that a group at a given time, is nothing over and above some individuals as they exemplify a certain complex condition.

\section{Hylomorphism and its limits}

On a hylomorphic mode of composition, at a given time, some material objects compose another as they stand in a certain relation to each other. There are rival accounts of hylomorphic composition in the market, but the theory of embodiments outlined by [Fine, 1999] appears tailor-made for our purpose. 4 There are two main ingredients to the account. According to Fine, some material objects $a, b, c, \ldots$ and a relation $R$ may be combined into a rigid embodiment where they are qualified by the relation $R$ : the rigid embodiment $a, b, c, \ldots / R$ only exists when the material objects $a, b, c, \ldots$ exist and are linked by the relation $R$. On this view, $a, b, c, \ldots$ are immediate material parts
${ }^{4}$ See [Koslicki, 2008] and [Goodman, 2016] for alternative versions of hylomorphism. 
of $a, b, c, \ldots / R$, and $R$ is a formal part of it: a water molecule consists of two hydrogen atoms $h_{1}, h_{2}$ and an oxygen atom $o$ linked by certain covalent bonds, $h_{1}, h_{2}, o / M$. The atoms $h_{1}, h_{2}, o$ are its immediate material parts, and the molecular structure $M$ is a formal part. Suits, clusters, and bouquets are further putative examples of rigid embodiments. In each case, a rigid embodiment consists of some objects, which we may call its immediate material parts, and the relation they exemplify. They are called rigid because they have their immediate material parts necessarily if at all. In this respect, rigid embodiments seem different from groups, which generally have their members only temporarily and contingently.

Groups are closer to what Fine calls variable embodiments. Given a condition $F$ that applies to exactly one material object at each given time, Fine writes / F/ for the variable embodiment that is manifested at a time by whatever exemplifies condition $F$ at it. 5 Given an appropriate choice of condition $F$, we may conceive of a committee as a variable embodiment, which at a given time $t$ consists of a rigid embodiment of individuals bound by condition $F$. This allows for other groups to be bound by less cohesive relations. The members of a queue are bound by a complex condition, which, at a given time, is satisfied by whatever individuals exemplify a certain spatial arrangement as they wait for a certain purpose.

The hylomorphic account of groups appears to fulfill many of our desiderata. Many groups have their members only temporarily and contingently. The members of a variable embodiment at a given time are the immediate material parts of the rigid embodiment that constitutes it at the time. But since one and the same variable embodiment may be constituted by different rigid embodiments at different times and worlds, there is room for groups to change their members across times and worlds.

Second, the account makes allowance for coincidence. Two rigid embodiments may at a given time, consist of exactly the same members and nevertheless remain different on account of the fact that they are unified by different relations. The search committee and the admissions committee may at a given time, be constituted by two rigid embodiments with the same members.

In the third place, the present account of what it is for an individual to be a member of a group at a given time appears to illuminate the distinction between highly structured groups like committees and courts and less cohesive groups like queues and multitudes. In order to be a member of a committee at a given time, one must stand in a rather complex relation to all the other individuals in a certain rigid embodiment, and the fact that they stand in that relation may be brought to bear on the question of whether they exhibit shared
${ }^{5}$ This is not very far apart from the view defended by [Ritchie, 2013]. Think of a variable embodiment as the realization of a structure, which is manifested at each time at which it exists by the occupants of different nodes in the structure. 
intentionality and agency. In contrast to this, in order to be a member of a queue at a given time, one need only stand in a certain spatial relation to the other individuals in the queue as they wait for a certain purpose. This relation, you may think, need not support the emergence of a shared intentionality or agency.

Last, but not least, the account may be supplemented with an account of atemporal mereological relations on the domain of rigid and variable embodiments. ${ }^{6}$ When properly fleshed out, this account should enable us to make a distinction between the variable embodiment parts of a variable embodiment and other variable embodiments that merely coincide with them.

But this is not the account we want to defend in this paper. One reason is that we still lack the means to do justice to the judgment that at a given time, a queue is nothing over and above some individual members as they stand in line for a certain purpose. This is what made the account outlined by [Simons, 1987, 4.4] quite attractive in the first place.

The other reason is a threat of inconsistency. Hylomorphic accounts of composition generally come with existence and identity conditions for rigid embodiments. The existence conditions specify the conditions under which some material objects linked by a relation give rise to a rigid embodiment. The identity conditions for rigid embodiments tell us what it takes to identify two rigid embodiments. The problem we have in mind is illustrated by the postulates found in [Fine, 1999]. The first postulate aligns with the thought that there are no restrictions on hylomorphic composition:

Rigid Existence: If some material objects $a, b, \ldots$ stand in relation $R$ at some world-time, then there is a rigid embodiment $a, b, \ldots / R$, which exists at a world-time if and only if $a, b, \ldots$ stand in $R$ at the world-time. ${ }^{7}$

The second postulate tells us that two rigid embodiments are the same if, and only if, they consist of the same individuals as they are bound by the same relation:

Rigid Identity: The rigid embodiment $a, b, \ldots / R$ is the same as the rigid embodiment $a^{\prime}, b^{\prime}, \ldots / R^{\prime}$ if and only if $a=a^{\prime}, b=b^{\prime}, \ldots$ and $R$ is the same as $R^{\prime} .{ }^{8}$

One consequence of the identity postulate is the following:

Rigid Extensionality: The rigid embodiment $a, b, \ldots / R$ is the same as the rigid embodiment $a^{\prime}, b^{\prime}, \ldots / R^{\prime}$ only if $a=a^{\prime}, b=b^{\prime}, \ldots$

The postulates turn out to be inconsistent with the existence of more than one material object. 9
${ }^{6}$ See [Fine, 1999] for an example of such an account.
7 Talk of world-times is shorthand for quantification over pairs of worlds and times.

${ }^{8}$ [Fine, 1999] acknowledges that the requirement that the relation $R$ be the same as $R^{\prime}$ makes rigid embodiments more fine-grained than one may like. He suggests we may instead require only that the state of affairs consisting of $a$ and $b$ as they stand in $R$ be the same as the state of affairs consisting of $a$ and $b$ as they stand in $R^{\prime}$.
${ }^{9}$ The two claims are consistent with the
existence of a single material object. For
given a singleton domain $\{a\}$, we may
let $a=a / R$ for every relation $R$.
Given more than one material object,
define a relation $E$ that relates $x$ and
$y$ at a world/time iff there are some
material objects $a, b, \ldots$ such that $x$ is
one of them and $y=a, b, \ldots / R$ for some
relation $R$. By existence, given some
objects $a, b, \ldots$, there is some $y$ such
that for every $x, x$ stands in $E$ to $y$ iff
$x$ is one of $a, b, \ldots$ This contradicts a
theorem of plural logic:
$\exists x \exists y x \neq y \rightarrow \neg \forall x x \exists y \forall x(R x y \leftrightarrow x \prec x x)$.

This theorem tells us that given two objects, it is not possible for a binary relation $R$ to inject pluralities into objects - or, given the usual gloss, there are more pluralities than objects. For discussion and a proof of the theorem, see [Florio, 2014, section 5]. 
We face a choice. We must weaken either the existence or the identity postulate. But while a restriction of the existence postulate may perhaps be motivated by common sense, it appears to lead to vague and intolerably arbitrary answers to the existence question. ${ }^{10}$ Maybe this is not, in the end, a decisive concern. ${ }^{11}$ But to the extent to which one is moved by arbitrariness concerns, one should look elsewhere for a response.

The other horn of the dilemma is to weaken the identity postulate in order to allow for the identification of rigid embodiments with different immediate material parts: one and the same rigid embodiment may be specified as $a, b, \ldots / R$ and $a^{\prime}, b^{\prime}, \ldots / R$, where $a, b, \ldots$ are different material objects from $a^{\prime}, b^{\prime}, \ldots$. This means that $a, b, \ldots / R$ exists at a world-time $w$ if and only if $a^{\prime}, b^{\prime}, \ldots / R$ does. What if $R$ is trivially satisfied by any objects provided they exist? We will find that different pluralities of material objects $a, b, \ldots$ and $a^{\prime}, b^{\prime}, \ldots$, will have to be intimately entangled in that they exist at exactly the same worldtimes. This might perhaps be tolerable if it could be a localized phenomenon. But it would unfortunately have to be quite widespread. ${ }^{12}$

\section{A Theory of Rigid Plural Embodiment}

The dilemma presupposes that embodiments are themselves material objects over and above their immediate material parts: a water molecule, we think, is a material object over and above two hydrogen atoms and an oxygen atom qualified by a certain molecular structure. But we now set out to make room for the concept of a rigid plural embodiment, one which consists of some objects linked by some plural condition, for example, two hydrogen atoms and one oxygen atom as they exemplify a certain molecular structure.

In what follows, we focus on the viability of an account of rigid plural embodiments. We aim to formalize the approach in an extension of plural logic. We will generally let plural variables, $m m$, to refer to some material objects, and we will consider plural conditions $A(\mathrm{~mm})$ these objects may satisfy. Some of them may include irreducibly collective predications such as " $m m$ are arranged in a circle" or " $m m$ are scattered". Two postulates give existence and identity conditions for rigid plural embodiments:

Rigid Plural Existence: If some material objects $m m$ satisfy a plural condition $A$ at a world-time, then there are some material objects $m m / A$, which exist at a world-time iff $m m$ satisfy $R$ at the worldtime.

Rigid Plural Identity: Some material objects, $m m / A$, are the same as some material objects, $n n / B$ if and only if a material object is
${ }^{10}$ This is part of the motivation [Fine, 1999] cites for the existence postulate.

${ }^{11}$ [Korman, 2010] responds to the argument from arbitrariness, and [Koslicki, 2008] builds a restriction of existence into her own version of hylomorphism. Moreover, [Fine, 2007] argues that some arbitrary restriction on principles like the rigid existence postulate becomes unavoidable when we consider their interaction with other sorts of objects

\footnotetext{
${ }^{12}$ Take a relation $D$ in which some objects stand if and only if they are all distinct from each other. If the domain of material objects is a set, then we are committed to the claim that distinct sets of material objects give rise to one and the same rigid embodiment under $D$. It is a consequence of the ZermeloKönig inequality that at least one rigid embodiment corresponds to as many different sets of material objects under $D$ as there are sets of material objects altogether. This seems a stiff price to pay in exchange for consistency.
} 
one of $m m$ if and only if it is one of $n n$ and necessarily, for any material objects $x x, A(x x)$ if, and only if, $B(x x)$.

Think of $m m / A$ as some material objects $m m$ as qualified by a plural condition $A$ - or some material objects $m m$ qua $A$, for short. ${ }^{13}$ For a concrete example, consider a rigid plural embodiment given by two hydrogen atoms and an oxygen atom qua linked by certain covalent bonds. ${ }^{14}$

Given some individuals, there is generally more than one rigid plural embodiment of which they are members. There is Alice, Beth, and Charles as they stand next to other, and there is Alice, Beth, and Charles as they remain in close proximity to each other. These rigid plural embodiments are different because they exemplify different modal profiles: they can remain in close proximity even if they do not stand next to each other. On the other hand, they must remain in close proximity to each other if they are to stand next to each other. There is a sense in which the first rigid plural embodiment is more fragile than the second: the second could exist without the first one, but not vice versa. As a limit case, there is what we may call the bare plurality of Alice, Beth, and Charles, which is a rigid plural embodiment that exists provided only that they exist. ${ }^{15}$

We may give a more precise characterization of the modal profile of a rigid plural embodiment. A function $f$ from world-times to sets of individuals in them is defined for a world-time $w$ if, and only if, $f(w) \neq \varnothing$. A rigid modal profile is a function $f$ from world-times to sets of individuals in them, which (i) is defined for some world-time, and (ii) outputs the same set of individuals at any world-time for which it is defined. ${ }^{16}$ A rigid modal profile $f$ corresponds to a rigid plural embodiment $m m / R$ if, and only if, $f$ maps a world-time $w$ to the set of members of $m m$ if, and only if, $m m$ satisfy condition $A$ at $w .{ }^{17}$ If plural conditions $A$ and $B$ are necessarily coextensive, then the rigid plural embodiments $\mathrm{mm} / A$ and $\mathrm{mm} / B$ exemplify one and the same rigid modal profile. ${ }^{18}$ Against this backdrop, the rigid existence and identity postulates come down to this:

Each rigid modal profile is exemplified by a unique rigid plural embodiment.

Matters are different for rival accounts of the identity conditions for rigid plural embodiments. Should you require more than mere necessary equivalence of conditions $A$ and $B$ for the two rigid plural embodiments to be the same, you would find that many different rigid plural embodiments could exemplify one and the same rigid modal profile. ${ }^{19}$

We regiment the account of rigid plural embodiments in a manysorted extension of plural quantified modal logic.
${ }^{13}$ The plural conception of rigid embodiments is not unlike a plural generalization of Fine's conception of qua-objects in [Fine, 1982]: qua-objects are objects under a certain description. The difference is that we now allow for some objects in the plural to be qualified by a plural condition.

${ }^{14}$ Note, however, that the thesis that the three atoms and the relevant molecular structure form a rigid plural embodiment is itself neutral with respect to whether we should think of the water molecule as a complex object over and above the rigid plural embodiment. Some philosophers, for example, [Merricks, 2001] and [van Inwagen, 1990], deny the existence of a wide range of composite objects and paraphrase unreflective talk of composites in terms of plural quantification over simples. Maybe they could be tempted to use of the concept of plural embodiment in order to make do without composite objects over and above their parts, but whatever the prospects of this move, it is beyond the remit of this paper.

${ }^{15}$ You may think of the bare plurality of Alice, Beth, and Charles as the rigid plural embodiment that consists of them as they exist and remain different from each other.

${ }^{16}$ More formally, $f$ is a rigid modal profile if, and only if, (i) $f(w) \neq \varnothing$ at some world $w$, and (ii) for any worldtimes $w$ and $w^{\prime}$, if $f(w) \neq \varnothing$ and $f\left(w^{\prime}\right) \neq \varnothing$, then $f(w)=f\left(w^{\prime}\right)$.

${ }^{17}$ Recall the convention to speak of the members of a plurality to mean the objects each of which is one of the objects in the plurality.

${ }^{18}$ As usual, $A$ and $B$ are necessarily coextensive, if necessarily, no matter what some $x x$ may be, $A(x x)$ if, and only if, $B(x x)$.

${ }^{19}$ More fine-grained accounts of rigid plural embodiments are the next step in the area. We will, however, take it one step at a time by seeking some assurance of the coherence of the coarse-grained account of rigid plural embodiments. 


\subsection{A Formal Framework}

Plural logic is regimented in a two-sorted first-order language with individual variables, $x, y, z, \ldots$, plural variables, $x x, y y, z z, \ldots$, and two primitive predicates: $x \prec y y$, read: " $x$ is one of $y y$ ", and $x=y$ is read: " $x$ is identical with $y$ ". In addition to this, we allow a stock of non-logical predicates into the language some of which take plural variables as arguments.

We want to extend the framework to allow for quantification over rigid plural embodiments - in addition to individuals and bare pluralities. Since bare pluralities are a special case of rigid plural embodiments, one may be tempted to keep the framework two-sorted and allow plural variables to range over rigid plural embodiments.

This choice would be parallel to the one made by theorists who regiment plural logic as a one-sorted framework. ${ }^{20}$ Since individuals are a special case of a plurality - a plurality of a single individual - and plural variables may be thought to range over individuals and pluralities. ${ }^{21}$ But while the simplicity of a one-sorted framework is sometimes attractive, a two-sorted framework still allows for a more perspicuous formalization of the axioms fo plural logic. And this, I think, explains the sociological fact that many still opt for a two-sorted formalization of plural logic.

In the case at hand, there is a similar trade off between the simplicity of a two-sorted framework and the lack of perspicuity of the corresponding regimentations of rigid plural existence and identity. ${ }^{22}$ So, we will reserve $x x, y y, z z, \ldots$ to range over bare pluralities and introduce a new style of plural variable, $\overline{x x}, \overline{y y}, \ldots$, for rigid plural embodiments. We supplement the new three-sorted language with another primitive predicate: $x \prec \overline{y y}$, read: " $x$ is one of $\overline{y y} . " 23$ It will be helpful to introduce two defined predicates: $\overline{x x} \ll \overline{y y}$ as an abbreviation for: $\forall x(x \prec \overline{x x} \rightarrow x \prec \overline{y y})$, and $\overline{x x} \equiv \overline{y y}$, as an abbreviation for: $\overline{x x} \ll \overline{y y} \wedge \overline{y y} \ll \overline{x x}$.

We take bare pluralities to be governed by the usual axioms of plural logic. In particular, we assume an axiom of plural comprehension:

$$
\exists x A(x) \rightarrow \exists x x \forall x(x \prec x x \leftrightarrow A(x)), \quad \text { (Comprehension) }
$$

provided $x x$ does not occur freely in $A(x)$. Given a condition $A$, there are some objects, which are all and only the objects that satisfy the condition. This is an important difference between pluralities and sets. While the condition non-self-membered determines a plurality of all and only non-self-membered objects, we know all too well that there is no set of them on account of Russell's paradox.

And we assume, as usual, that bare pluralities are extensional:

$$
x x \equiv y y \rightarrow(A(x x) \leftrightarrow A(y y)) . \quad \text { (Extensionality) }
$$

${ }^{20}[$ McKay, 2006] is a case in point.

\footnotetext{
${ }^{21}$ [McKay, 2006, Chapter 13, section 3] outlines this route in quite some detail. His regimentation of plural logic begins with the atomic predicate $x x \ll y y$, read: " $x x$ are among $y y$ " and defines a plural predicate $I x x$, read: " $x x$ are one in number," as an abbreviation for: $\forall y y(y y \ll x x \rightarrow x x \ll y y)$. One is then in a position to introduce a "one of" predicate in terms of "among" and proceed to formulate the usual principles of plural logic in a one-sorted framework.

${ }^{22}$ Indeed, since individuals themselves are special cases of bare pluralities and bare pluralities are rigid plural embodiments, we could in principle make do with a one-sorted framework, though one that is not particularly perspicuous.

${ }^{23}$ We take "one of" to be implicitly relativized to world-times.
} 
This is motivated by the thought that bare pluralities are completely determined by its members. One may then define a plural identity predicate $x x==y y$ to abbreviate: $x x \equiv y y$.

Unlike bare pluralities, plural embodiments are not extensional. Alice, Beth, and Charles as they stand next to each other share all their members with Alice, Beth, and Charles as they talk to each other, but the two rigid plural embodiments remain different as they exemplify different modal profiles. So, we cannot define quasi-plural identity, $\overline{x x}==\overline{y y}$, in terms of $\overline{x x} \equiv \overline{y y}$. Instead, we will take the identity $==$ predicate for rigid plural embodiments to be primitive and and governed by a quasi-plural indiscernibility principle. ${ }^{24}$

$$
\overline{x x}==\overline{y y} \rightarrow(A(\overline{x x}) \leftrightarrow A(\overline{y y})) . \quad \text { (Indiscernibility) }
$$

In order to regiment the rigid plural existence and identity postulates, we supplement the framework with a modal operator $\square$ for metaphysical necessity governed, as usual, by the axioms of the minimal normal modal logic K. Now, admittedly, the simplest modal plural quantificational logic — with identity - and necessitation allows one to derive instances of what is sometimes known as the "Necessary Necessity of Existence":

$$
\square \forall \alpha \square \exists \beta \alpha=\beta
$$

where $\alpha$ and $\beta$ stand for variables of the same sort. ${ }^{25}$ When we substitute plural variables for $\alpha$ and $\beta$, we have the claim that necessarily, no matter what some objects may be, they exist necessarily. ${ }^{26}$

Some embrace the derivability of (NNE) as evidence for necessitism - the view that necessarily, every object exists necessarily. But even necessitists should acknowledge a difference between objects that enjoy the sort of concrete existence exemplified by ordinary blades and handles and the merely possible knives that could have been built from them. In this vein, they may attempt to do justice to ordinary judgments of existence by means of a stock of concrete existence predicates $E \alpha$ for each style of variable. This is, incidentally, why (NNE) need not wreak havoc with the thought that a plural rigid embodiment $m m / A$ may not exist at a world-time $w$ if $m m$ fail to satisfy the plural condition $A(x x)$ at $w$ : a plural rigid embodiment consisting of a blade and a handle as they are joined together will at $w$ not enjoy the same concrete existence as the relevant blade and handle unless they are in fact joined together at $w$. A necessitist will simply recast the thought as one to do with concrete existence: the rigid plural embodiment exists necessarily, but it does not necessarily enjoy concrete existence.

Contingentism is the denial of necessitism - the view that pos-
${ }^{24}$ The mere addition of quasi-plural variables is no obstacle for consistency: to construct a model we may simply let quasi-plural variables range over the same domain as the original plural variables. Even if you take the new variables to be governed by the nonextensional fragment of plural logic, the key observation is that any model of the classic logic of plurals is itself a model of the non-extensional fragment.

${ }^{25}$ Since $\exists \beta \alpha=\beta$ is a theorem for each style of variable, necessitation delivers $\square \exists \beta \alpha=\beta$ and the appropriate rule of universal generalization yields $\forall \alpha \square \exists \beta \alpha=\beta$. We obtain (NNE) by another application of necessitation.

${ }^{26}$ Necessitists generally adopt a constant domain model theory for quantified modal logic that validates all instances of the Converse Barcan (CBF) and the Barcan (BF) schemata:

$$
\begin{aligned}
& \square \forall \alpha A(\alpha) \rightarrow \forall \alpha \square A(\alpha) \\
& \forall \alpha \square A(\alpha) \rightarrow \square \forall \alpha A(\alpha),
\end{aligned}
$$

where $\alpha$ and $\beta$ stand again for variables of the same sort. 
sibly some objects do not exist necessarily. Many contingentists reject classical quantificational logic in response to the derivability of (NNE): they often weaken universal instantiation and introduce existence predicates $E \alpha$ for each style of variable to align with the range of the relevant quantifier: $\exists \beta \alpha=\beta .{ }^{27}$ Even if there are handles and blades, there are no merely possible knives, which could have - but are never - built from them.

But even contingentists fall back into talk of merely possible knives, and more importantly, many of these superficially problematic claims appear to track and communicate sensible modal truths. The official line of course is to disavow the literal truth of such statements and to hope to eventually be able to dispense with them with the help of a systematic method of paraphrase. ${ }^{28}$ In the meantime, we may grant contingentists a distinction between inner or actualist quantifiers governed by free quantificational logic and outer or possibilist quantifiers governed by classical quantificational theory. ${ }^{29}$ Inner quantifiers track the domain of what exists, and outer quantifiers are used to simulate talk of merely possible objects.

Necessitists and contingentists agree that to exist in the most general sense of the word is to be something. But some sever the link between quantification and existence. Socrates, they say, is something, but he no longer exists since he has been dead for a while now. On this view sometimes known as noneist, (NNE) is still a theorem, just not a statement of the necessary necessity of existence. [Lewis, 1990] and [Priest, 2005] discuss similar positions. This provides yet a different interpretation of the formalism on which the most general sense of existence is to be tracked by means of the existence predicates as opposed to quantification and identity.

The debate between necessitism and contingentism is orthogonal to the viability of an account of rigid plural embodiments. In what follows, we adhere to classical quantificational logic with identity and introduce a stock of existence predicates $E \alpha$ for each style of variable. If you are a necessitist, read $E \alpha$ as: " $\alpha$ has concrete existence". On the other hand, if you are a contingentist, read the quantifiers to be outer quantifiers designed to simulate quantification over merely possible objects and think of $E \alpha$ as the broadest existence predicate, which, for you, aligns with the range of the inner quantifiers. ${ }^{30}$

Whatever your sympathies, you will presumably take $E$ to be governed by a heredity principle according to which that the existence of a bare plurality or a rigid plural embodiment entails the existence of each individual member:

$$
\square(E \alpha \rightarrow \forall x(x \prec \alpha \rightarrow E x))
$$

where $\alpha$ is a placeholder for either a plural or quasi-plural variable.

\footnotetext{
${ }^{27}$ Universal instantiation is the axiom: $\forall \alpha \phi \rightarrow \phi[\beta / \alpha]$ in which $\beta$ is supposed to be free for $\alpha$ in $\phi$. [Cresswell and Hughes, 2012] outline some restrictions of the principle that would enable contingentists block the derivation of $(\mathrm{CBF})$ and (NNE) as well as the validity of (BF).
}

\footnotetext{
${ }^{28}$ According to [Fritz and Goodman, 2017], the prospects for a completely general method of paraphrase are bleak, since the best proposals rely on substantial assumptions that are in tension with other contingentist tenets.

${ }^{29}$ [Bricker, 1989] provides a concrete example of how contingentists can make profitable use of outer or possibilist quantifiers for certain theoretical purposes.
}

$3^{3}$ In what follows, I will simply read
$E \alpha$ as " $\alpha$ exists" with the understanding
that necessitists and contingentists will
interpret it as concrete and absolute
existence respectively. 
Two rigidity axioms describe a feature bare pluralities and rigid plural embodiments have in common:

$$
\begin{aligned}
& x \prec \alpha \rightarrow \square(E \alpha \rightarrow x \prec \alpha) \\
& x \nprec \alpha \rightarrow \square(E \alpha \rightarrow x \nprec \alpha)
\end{aligned}
$$

Bare pluralities cannot change their members: if Alice is one of some people, then necessarily, she is one of them, if they exist. And if she is not one of them, then she can never be one of them. But rigid plural embodiments share this feature: if Alice is one of Alice, Beth, and Charles as they stand next to each other, then necessarily, if they she is necessarily one of them as they stand next to each other. And if she is not on of them as they stand next to each other, then she can never be one of them as they stand next to each other.

One key difference between bare pluralities and rigid plural embodiments is that they come with different existence conditions. While a bare plurality exists at a world-time if, and only if, the members do, there is no guarantee that a rigid plural embodiment should exist at a world-time even if the members do: Alice, Beth, and Charles can exist at a world-time even if the rigid plural embodiment of Alice, Beth, and Charles as they stand next to each other fails to exist. In symbols, we endorse another heredity principle:

$$
x x \equiv \alpha \rightarrow \square(E \alpha \rightarrow E x x) .
$$

But we cannot reverse the conditional embedded in the consequent.

We can finally offer formal regimentations of the plural existence and identity postulates. Here is a schematic formalization of rigid plural existence:

$$
\begin{array}{r}
(E x x \wedge A(x x)) \rightarrow \exists \overline{x x}(\overline{x x} \equiv x x \wedge \square(E \overline{x x} \leftrightarrow \\
(E x x \wedge A(x x))))
\end{array}
$$

(R-Existence)

To make it vivid, consider a non-distributive plural condition like $x x$ are arranged in a circle. According to (R-Existence), if some objects exist and they are arranged in a circle, there are some objects-which are a rigid plural embodiment-with the same members as them, and which, necessarily, exist if, and only if, each of them exists and they are arranged in a circle. ${ }^{31}$

We individuate rigid plural embodiments by their existence conditions: in particular, we identify rigid plural embodiments with the same existence conditions:

$$
\overline{x x}==\overline{y y} \leftrightarrow \overline{x x} \equiv \overline{y y} \wedge \square(E \overline{x x} \leftrightarrow E \overline{y y}) \quad \text { (R-Identity) }
$$

The combination of (R-Existence) and (R-Identity) enables us to define the rigid plural embodiment $x x / A$ as the unique rigid embodiment $\overline{x x}$ coextensive with the bare plurality $x x$, that is, $\overline{x x} \equiv x x$,
${ }^{31}$ Notice the implicit role of $(\mathrm{R})$ in the regimentation of the existence postulate. Without it, (R-Existence) cannot guarantee that $\overline{x x}$ has any members at worlds other than the world of evaluation. 
whose existence conditions require $x x$ to exist and satisfy condition $A$, that is, $\square(E \overline{x x} \leftrightarrow(E x x \wedge A(x x))) 3^{32}$

Given this definition, as a special case of (R-Identity), we find:

$$
x x / A==x x / B \leftrightarrow \square(A(x x) \leftrightarrow B(x x))
$$

It is a consequence of (R-Identity) that given some material objects $x x, x x / A$ and $x x / B$ are one and the same rigid plural embodiment if $A$ and $B$ are necessarily coextensive conditions.

The theory of rigid plural embodiments is consistent: to find a model, we can take a variable domain model for quantified plural modal logic and let plural variables of different sorts range over rigid modal profiles. The only difference is that $x x, y y, \ldots$ range over a special class of rigid modal profiles, which assign the same set $S$ of individuals to every world $w$, whose inner domain includes $S$.

\subsection{Interlude: A Russellian Puzzle}

There is still an inconsistent position in the vicinity. If a material object $m$ exemplifies condition $F$ at a given world-time, then $m / F$ is a qua object which exists at a given world-time if and only if $m$ exemplifies $F$ at the given world-time. 33 And qua-objects may be governed by singular counterparts of the rigid plural existence and identity postulates:

Qua Existence: If a material object $m$ instantiates $F$ at a world-time $w$, then there is some object $m / F$, which exists at $w$ if, and only if, $m$ instantiates $F$ at $w$.

Qua Identity: $m / F=n / G$ if and only if $m=n$ and $F$ and $G$ are necessarily coextensive.

But these two principles leave us again on the brink of inconsistency. In fact, we need only a minimal consequence of the second postulate:

Qua Extensionality: $m / F=n / G$ only if $m=n$ and $F$ and $G$ are coextensive. 34

This observation is due to [Fairchild, 2017]. Say an object $m$ instantiates its own gloss if, and only if, there is some $n$ and $F$ such that (i) $m=n / F$, and (ii) $m$ instantiates $F$. Now, let $R$ be the property a material object $m$ instantiates if, and only if, $m$ does not instantiate its own gloss. Given more than one material object, there is at least one object $m$ that instantiates $R$. By Qua Existence, there is a qua-object $m / R$. But this turns out to be inconsistent with Qua Extensionality.35

It is instructive to check how the problem is avoided in the present framework. We regiment Qua Existence by means of a particular
${ }^{32}$ Given a definite descriptor operator l, we may think of $x x / A$ as: $\iota \overline{x x} \forall x(x \prec$ $\overline{x x} \leftrightarrow x \prec x x) \wedge \square(E \overline{x x} \leftrightarrow(E x x \wedge$ $A(x x)))$. The thought, as usual, is that we can uniformly eliminate every occurrence of $x x / A$ in the formal framework in terms of the relevant definite description. ${ }^{33}$ The concept of a qua-object can be
traced back to [Fine, 1982].

${ }^{34}$ Compare this with the rigid extensionality postulate we exploited in section 2 to develop a problem for the theory of rigid embodiment.

${ }^{35}$ See [Fairchild, 2017, section 1] for detailed argument. She points out that attractive as the extensionality principle may seem, it is not forced upon one by the hylomorphic picture that underlies the existence postulate for qua objects. 
instance of (R-Existence):

$$
\begin{aligned}
(E x \wedge A(x)) \rightarrow \exists \overline{x x}( & \forall x(x \prec \overline{x x} \leftrightarrow x=m) \wedge \\
\square(E \overline{x x} \leftrightarrow(E x \wedge A(x)))) & \text { (Q-Existence) }
\end{aligned}
$$

But the key observation is that there is no reason to expect the relevant qua-object to figure in the range of individual variables. The rigid plural embodiment $x / A$ generated by (Q-Existence) from a material object $x$ and a condition $A$ may itself not be a material object in the range of individual variables. ${ }^{36}$ This is parallel to the fact that a rigid plural embodiment of two hydrogen atoms and an oxygen atom as linked by certain covalent bonds does not itself lie in the range of an individual variable.

Unlike (Q-Existence), the main effect of (R-Existence) is to generate an additional layer of rigid plural embodiments over an initial domain of material objects - in much the way in which plural comprehension only guarantees a layer of bare pluralities over an antecedently given domain of individuals. 37 So, to the extent to which a water molecule is a material object, it is not to be identified with the rigid plural embodiment that consists of two hydrogen atoms and an oxygen atom as linked by certain covalent bonds. Nor is it clear that rigid plural embodiments can themselves be parts of the material objects of which molecules are parts. This may itself be regarded as a bound on the scope of the theory of plural embodiments, but remember it was never meant to take the place of a hylomorphic account of material objects.

The concern may arise at this point that we never have an opportunity to iterate the formation of plural embodiments out of antecedently given plural embodiments. One may perhaps complain that in contrast to this, a perfectly general account of groups ought to allow for a measure of iteration: even if different faculty members compose different committees, there is no reason to bar the existence of a higher-level rigid plural embodiment of two committees as they collaborate to a advance a common purpose. Maybe committees of committees consist at a given world-time, of such higher-level rigid plural embodiments.

There are two avenues of response available to us, one more conservative than the other. The more conservative response is to insist pace [Uzquiano, 2004], for example, that the problem is only apparent. For you may plausibly take a committee of committees to consist at a given world-time of some individual representatives or proxies for certain committees and you may take their remit at the relevant world-time to be the coordination the committees they represent.

The more ambitious response is to expand the account of rigid embodiments into a hierarchy of levels that allows for a measure of 
iteration. Some philosophers think that there is a form of quantification that stands to plural quantification much like plural quantification stands to singular quantification. ${ }^{38}$ We may following [Linnebo, 2017], call this style of quantification second-level plural quantification. More generally, these philosophers suggest we may attempt to generalize this style of quantification to every finite level to obtain what is for all intents and purposes a version of finite type theory. [Rayo, 2006] has indeed developed a formal framework for extensions of plural quantification to finite levels and [Linnebo and Rayo, 2012] have more recently expanded the framework to allow for transfinite levels. Although we cannot hope to adjudicate the issue in this paper, we note that it should be quite simple to supplement the version of finite type theory to include quantification over generalizations of rigid plural embodiments for each finite level. This would certainly be able to accommodate higher-level rigid plural embodiments of the sort mentioned above.

\subsection{Rigid Plural Embodiments and their Parts}

We relativized the relation an individual bears to a rigid plural embodiment of which it is a member to a world-time. But we can still ask whether rigid plural embodiments enter into atemporal mereological relations. Let us begin with the question of what is for a rigid plural embodiment to be part of another. One may tentatively answer that $m m / A$ is part of $n n / B$ if, and only if, part of what it is for $n n$ to satisfy $B$ is for $m m$ to satisfy $A .39$ On the present framework, the satisfaction of conditions $A$ and $A$ by $n n$ and $m m$ is intimately connected to the existence conditions of $\mathrm{mm} / A$ and $n n / B$. This suggests the postulate:

Rigid Plural Parthood: The rigid embodiment $m m / A$ is part of the rigid embodiment $n n / B$ if, and only if, $n n / B$ exists at a worldtime $w$ only if $m m / A$ does.

More generally, if $f_{A}$ and $f_{B}$ are the modal profiles of two rigid plural embodiments $m m / A$ and $n n / B$, respectively, $m m / A$ is part of $n n / B$ if, and only if, for every world-time $w, f_{B}(w) \subseteq f_{A}(w)$.

In the special case in which two rigid plural embodiments are of the form $x x / A$ and $x x / B$, the less fragile plural embodiment will be part of the more fragile one. By way of illustration, consider a case in which Alice, Beth, and Charles line up at the box office, and compare the rigid plural embodiments specified below:

- Alice, Beth, and Charles as they line at the box office $(a, b, c / A) .4^{\circ}$

- Alice, Beth, and Charles as they stand close to each other $(a, b, c / B)$.

- Alice, Beth, and Charles as distinct from each other $(a, b, c / C)$.
${ }^{38}$ The thought is explored, for example, in [Hazen, 1993], [Rayo, 2006], and [Linnebo and Nicolas, 2008]

\footnotetext{
39 This is in line with the account outlined in [Fine, 1999]:

Rigid Parthood: The rigid embodiment $\left(x_{1}, x_{2}, \ldots\right) / R$ is part of the rigid embodiment $\left(y_{1}, y_{2}, \ldots\right) / S$ if part of what it is for $y_{1}, y_{2}, \ldots$ to stand in relation $S$ is for $x_{1}, x_{2}, \ldots$ to stand in relation $R$.
}

${ }^{40}$ We use list terms of the form $a, b, c$ as plural expressions for the bare plurality whose members are exactly $a, b$, and $c$. 
Here we find three different rigid plural embodiments with different existence conditions. They have been listed in order from more to less fragile rigid plural embodiments; the last one is just the bare plurality of Alice, Beth, and Charles. It follows that the last two rigid plural embodiments are parts of their predecessors in the order. ${ }^{41}$

In the formal framework, we can articulate the thought as followswhere $\alpha$ again is a placeholder for a plural or quasi-plural variable:

$$
\alpha \leq_{R} \beta \leftrightarrow \alpha \ll \beta \wedge \square(E \beta \rightarrow E \alpha)
$$

Notice that we cannot do without the first conjunct. Two rigid plural embodiments may satisfy the second conjunct even if one is not part of the other: necessarily, the bare plurality of Alice and Beth exist only if the bare plurality of their singletons do, but we may not want to allow the bare plurality of singletons of Alice and Beth to count as part of the bare plurality of Alice and Beth.

Given $\left(\leq_{R}\right)$, we are in a position to derive the axioms of core mereology. Reflexivity and Transitivity fall out as immediate consequences of the definition:

Reflexivity: $\alpha \leq_{R} \alpha$

Transitivity: $\alpha \leq_{R} \beta \wedge \beta \leq_{R} \gamma \rightarrow \alpha \leq_{R} \gamma$

For Reflexivity, it is trivial to note that $\alpha \ll \alpha$ and of course $\square(E \alpha \rightarrow$ $E \alpha)$. Transitivity is a simple consequence of the transitivity of $\ll$ and the definition: if $\alpha \leq_{R} \beta$ and $\beta \leq_{R} \gamma$, then since $\alpha \ll \beta$ and $\beta \ll \gamma$, we have $\alpha \ll \gamma$. Moreover, since $\square(E \gamma \rightarrow E \beta)$ and $\square(E \beta \rightarrow E \alpha)$, we have that $\square(E \gamma \rightarrow E \alpha)$. It follows that $\alpha \leq_{R} \gamma$.

The status of anti-symmetry is closely linked to the status of ( $R$ Identity). The latter principle guarantees the truth of all instances of anti-symmetry where $\alpha$ and $\beta$ are plural variables of either sort:

$$
\text { Anti-symmetry: } \alpha \leq_{R} \beta \wedge \beta \leq_{R} \alpha \rightarrow \alpha==\beta
$$

If $\alpha$ and $\beta$ are mutual parts, then given $\left(\leq_{R}\right)$, they are coextensive, that is, $\alpha \equiv \beta$. Moreover, given $\left(\leq_{R}\right)$ again, $\alpha$ and $\beta$ enjoy the same existence conditions, that is, $\square(E \beta \leftrightarrow E \alpha)$. It follows from (R-Identity) that $\alpha==\beta$.

We now define proper part, $\alpha<_{R} \beta$, and overlap, $\alpha \circ_{R} \beta$, as usual in terms of part and identity:

$$
\begin{array}{r}
\alpha<_{R} \beta:=\alpha \leq_{R} \beta \wedge \neg \alpha==\beta \\
\alpha \circ_{R} \beta:=\exists \gamma\left(\gamma \leq_{R} \alpha \wedge \gamma \leq_{R} \beta\right)
\end{array}
$$

We formulate a principle of weak supplementation as usual:

$$
\text { Weak Supplementation: } \alpha<_{R} \beta \rightarrow \exists \gamma\left(\gamma \leq_{R} \beta \wedge \neg \gamma \circ_{R} \alpha\right) \cdot 4^{2}
$$

${ }^{41}$ More formally, let $f_{A}, f_{B}$, and $f_{C}$ the modal profiles corresponding to each rigid plural embodiment. Since Alice, Beth, and Charles can only line up at the box office if they stand close to each other, we have that the second is part of the first - for each $w, f_{B}(w)$ is a subset of $f_{A}(w)$. Moreover, the first is not part of the second: Alice, Beth, and Charles could at a world-time $w$, stand close to each other and not line up at the box office. This would be a case in which $f_{A}(w)=\varnothing$, whereas $f_{B}(w)=\{a, b$, c\}. So, it is not the case that for each $w, f_{A}(w)$ is a subset of $f_{B}(w)$. Since for each $w, f_{A}(w) \subseteq f_{B}(w) \subseteq f_{C}(w)$, the bare plurality of Alice, Beth, Charles is indeed part of the other two rigid plural embodiments.

\footnotetext{
${ }^{42}$ For discussion of supplementation principles and the axioms of mereology more generally, see [Simons, 1987] and [Varzi, 2016].
} 
However, there is no reason to expect rigid plural embodiments to be bound by this principle. To return to an earlier example, the bare plurality of Alice, Beth, and Charles is part of more fragile rigid plural embodiments that consist of them as they satisfy a certain condition. The bare plurality of Alice, Beth, and Charles is a proper part of the rigid plural embodiment that consists of them as they stand next to each other. But notice that any rigid plural embodiment that is part of the latter will have to contain some of Alice, Beth, and Charles as members. So, they will have a bare plurality of them as a part and this part will certainly overlap the bare plurality of Alice, Beth, and Charles.

A supplementation principle is still available when we restrict attention to bare pluralities. Unlike other rigid plural embodiments, bare pluralities are governed by the heredity principle:

$$
x x \ll y y \rightarrow \square(E y y \rightarrow E x x)
$$

Given $\left(\leq_{R}\right)$ and $\left(\mathrm{H}_{\ll}\right)$, the restriction of $\leq_{R}$ to bare pluralities collapses into the relation $\ll$. In other words:

$$
x x \leq_{R} y y \leftrightarrow x x \ll y y
$$

But when restricted to bare pluralities, $\ll$ is governed by the axioms of classical extensional mereology.43 In particular, bare pluralities satisfy a principle of strong supplementation:

$$
\text { Strong Supplementation: } x x \not_{R} y y \rightarrow \exists z z\left(z z \leq_{R} x x \wedge \neg z z \circ_{R} y y\right)
$$

For suppose $x x \not_{R} y y$. This means that $\exists x(x \prec x x \wedge \neg x \prec y y)$. By (Comprehension), $\exists z z \forall x(x \prec z z \leftrightarrow x \prec x x \wedge \neg x \prec y y)$. But it is now trivial to check that given such $z z, z z \leq_{R} x x$ and $\neg z z \circ_{R} y y .44$

By appeal to (Comprehension), we are in a position to derive a principle of unrestricted fusion for bare pluralities:

$$
\begin{aligned}
& \text { Unrestricted Fusion: } \exists x x \varphi(x x) \rightarrow \exists x x\left(\forall y y \left(\varphi(y y) \rightarrow y y \leq_{R}\right.\right. \\
& \left.x x) \wedge \forall y y\left(y y \leq_{R} x x \rightarrow \exists z z\left(\varphi(z z) \wedge y y \circ_{R} z z\right)\right)\right)
\end{aligned}
$$

For suppose some $x x$ are such that $\varphi(x x)$. By (Comprehension) again, $\exists u u \forall x(x \prec u u \leftrightarrow \exists y y(\varphi(y y) \wedge x \prec y y))$. Fix $u u$ as the objects in question. If some $y y$ are such that $\varphi(y y)$, then of course $y y \ll u u$. Moreover, if some $y y$ are such that $y y \ll u u$, then there are some $z z$ such that $\varphi(z z)$ and for some $x, x \prec z z$ and $x \prec y y$.

We cannot expect $\leq_{R}$ to be bound by classical mereology once we lift the restriction to bare pluralities: weak supplementation fails for rigid plural embodiments. It is natural to ask whether we have a fusion principle for them. We begin with two comments.

The first observation is that (Comprehension) is not enough to justify the existence of fusions of rigid plural embodiments. For
${ }^{43}$ This fact is proved and discussed by [McKay, 2006, Chapter 6].

\footnotetext{
${ }^{44}$ The contrast between rigid plural embodiments and bare pluralities is closely aligned with the contrast [Goodman, 2016] draws between material objects and matter. While matter verifies the axioms of classical extensional mereology, there is no reason to expect material objects to verify weak supplementation: the statue includes the clay as a proper part, but every part of the statue overlaps the clay. And this is perfectly compatible with the assumption that parthood is reflexive, transitive, and anti-symmetric on the domain of material objects. In particular, there is no reason to think that the statue is part of the clay even if the clay is part of the statue.
} 
a simple example, consider two rigid plural embodiments of the form $x x / A$ and $y y / B$. By (Comprehension), there is a unique bare plurality $x x+y y$, which fuses $x x$ and $y y .45$ But there is no guarantee that the bare plurality $x x+y y$ should include either rigid plural embodiment $x x / A$ or $y y / B$ as parts: maybe there is a world-time $w$ at which $x x+y y$ exist but neither $x x$ satisfies $A$ nor does $y y$ satisfy $B$.

Even if $x x+y y$ fails to include $x x / A$ and $y y / B$ as parts, there is a rigid plural embodiment $x x+y y / C$ based on $x x+y y$, which includes the other two as parts. ${ }^{46}$ This is a rigid plural embodiment, which consists of $x x+y y$ as they fuse $x x$ and $y y$ and $x x$ are $A$ and $y y$ are $B$; its existence at a world-time at which the other two exist, however, is guaranteed by (R-Existence).

The second comment is that absent supplementation, there is no hope for uniqueness. The rigid plural embodiment that consists of $x x+y y$ as they fuse $x x$ and $y y$ and $x x$ are $A$ and $y y$ are $B$ is only one fusion of the initial rigid plural embodiments but there are many others. In general, if $S$ is some formula such that

$$
\square(S \rightarrow A(x x) \wedge B(y y)),
$$

then by (R-Identity), there is a rigid plural embodiment, which consists of $x x+y y$ as they are such that $S$. But there will generally be choices of $S$, which entail but are not entailed by the conjunction $A(x x) \wedge B(y y) .47$ Clearly, $x x / A$ and $y y / B$ are parts of $x x+y y / S$. And for a rigid plural embodiments $\overline{z z}$ to be part of $x x+y y / S$, it must overlap at least one of $x x / A$ and $y y / B$.

This suggests an informal line of argument for unrestricted fusion for rigid plural embodiments:

$$
\begin{aligned}
& \text { Unrestricted Fusion: } \exists \alpha \varphi(\alpha) \rightarrow \exists \alpha(\forall \beta(\varphi(\beta) \rightarrow \beta \leq \alpha) \wedge \forall \beta(\beta \leq \\
& \alpha \rightarrow \exists \gamma(\varphi(\gamma) \wedge \beta \circ \gamma)))
\end{aligned}
$$

For suppose some rigid plural embodiment $\alpha$ satisfies condition $\varphi$ at a world-time $w$. And consider the fusion of bare pluralities coextensive with a rigid plural embodiment for which the condition obtains. This fusion exists by unrestricted comprehension for bare pluralities. Consider the fusion of the bare pluralities coextensive with a rigid plural embodiment of the relevant sort, and find a condition that is only satisfied in the world-time of evaluation. $4^{8}$ There is, by (R-Existence), a rigid plural embodiment which consists of the members of this bare plurality as the relevant condition is satisfied. This rigid plural embodiment is extremely fragile as it only exists at the world-time of evaluation, but this fragility is precisely what accounts for the fact that it includes as parts every rigid plural embodiment of the relevant sort.
${ }^{45}$ Since classical extensional mereology is true of bare pluralities, we are free to introduce $x x+y y$ to refer to the unique fusion of the two bare pluralities. By (Comprehension), $\exists z z \forall x(x \prec z z \leftrightarrow x \prec$ $x x \vee x \prec y y)$. This is a bare plurality that includes every member of $x x$, every member of $y y$, and nothing that is not a member of $x x$ or a member of $y y$.

${ }^{46}$ Let $C(u u)$ be the plural condition: $x x \ll u u \wedge y y \ll u u \wedge A(x x) \wedge B(y y)$.

$47 S$ could be the conjunction of $A(x x) \wedge$ $B(y y)$ with some merely contingent truth.

\footnotetext{
${ }^{48}$ One may be able to turn this into a formal argument in a higher-order framework in which we have propositional variables of the form $X, Y, Z, \ldots$ which correspond to o-place predicates to which we may assign a proposition. So, if $u u$ is the fusion fo the bare pluralities coextensive with a rigid plural embodiment that satisfies the condition, then we want to look at $u u / X$ under an assignment on which $X$ is mapped to a world-time proposition that is true at exactly $w$.
} 


\section{A Theory of Variable Plural Embodiments}

Groups are not rigid plural embodiments, but they are nevertheless constituted by them at each world-time at which they exist. A committee consists at a given world-time, of some individuals united by a common purpose, a principle of organization, and a set of conventional rules and procedures - a rigid plural embodiment. The individual members are a manifestation of the complex plural condition we associate with a committee.

To accommodate groups, we require the concept of a variable plural embodiment. This is a plural generalization of the concept of variable embodiment as originally introduced by [Fine, 1999]. Given a functional principle of generation $F$, which assigns at most one material object to a world-time $w$, we may consider a variable embodiment $/ F /$, which is a material object constituted at a given world-time $w$, by whatever object $F$ assigns to $w, F(w) .49$ [Fine, 1999] suggests a river, for example, is a variable embodiment generated by a principle of generation which picks out a portion of water at a given time..$^{0}$

We can generalize the account of plural embodiments to include variable plural embodiments. Call a plural condition $F$ functional if, and only if, it is satisfied by at most one bare plurality $\mathrm{mm}$ at each world-time $w$. Such a functional condition provides a principle of generation for a variable plural embodiment. We may conceive of the queue at the box office as a variable plural embodiment, whose manifestation at a given world-time $w$ is given by the individuals that exemplify the functional condition in question. Moreover, at $w$, we may take the queue to be constituted by the individuals as they manifest the functional condition associated with the queue.

The difference between a queue and a committee is largely due to the complexity of the functional condition they involve: in the case of a committee, the principle of generation consists of a principle of organization in addition with a set of rules and procedures and a certain purpose. In general:

Variable Plural Existence: If $F$ is a functional plural condition, then there is a variable embodiment $/ F /$, which exists at a world-time if, and only if, $F$ is satisfied by at most one bare plurality $m m$ of material objects at the world-time. Moreover, $/ F /$ is, at a worldtime $w$, constituted by the rigid embodiment $m m / F$, which consists of the individuals $m m$ that $F$ map to $w$ as they exemplify condition $F$ at the world-time in question.

This leaves open of course what are the identity conditions for variable plural embodiments. For a simple suggestion, consider:
${ }^{49}$ In more precise terms, [Fine, 1999] includes a principle of variable existence:

Variable Existence: If $F$ is a functional principle, then there exists a variable embodiment $/ F /$, which, at a given time $t$, exists iff some individual material object, $F(t)$, is the manifestation of the condition $F$ at $t$ in which case it constitutes the variable embodiment at $t$.

${ }^{50}$ Or one may conceive of a car as a variable embodiment generated by a functional principle that maps a time to a rigid embodiment of certain car-parts as they stand in an appropriate relation. 
Variable Plural Identity: A variable plural embodiment / F/ is the same as a variable plural embodiment / $G$ / if and only if $F$ is the same principle of generation as $G$.

The reason membership to a group / F/ may be temporary or contingent is that its principle of generation $F$ may assign different individuals to different world-times. The queue at the box office has many of its members only temporarily and contingently because it maps different bare pluralities to different world-times.

As for coincidence, two different groups / F/ and / G/ may remain different even if their respective principles of generation happen to assign the very same individuals to a given world-time $w: \mathrm{mm}$ are both the members of $F(w)$ and $G(w)$. This would be a case in which $/ F /$ and $/ G /$ would be constituted by two coextensive rigid plural embodiments, that is, $m m / F$ and $m m / G$ respectively. These rigid plural embodiments would still differ in virtue of the fact that they have a different modal profile.

By way of illustration, the search and the graduate committee are guided by different purposes, a different principle of organization, and a different set of rules and procedures. But their respective principles of generation may be satisfied by the same bare plurality of individuals at a given world-time $w$ : at $w$, they would be constituted by rigid plural embodiments with the same individual members. ${ }^{51}$

One key difference between variable and rigid plural embodiments has to do with the modal profiles they exemplify. A functional plural condition $F$ maps world-times to sets of individuals that exist at those world-times, that is, the set of individuals that satisfy the condition in question. Call a function $F$ from world-times to sets of objects that exist at those world-times a variable modal profile if, and only if, for some $w, F(w) \neq \varnothing$. To each variable modal profile $F$, there corresponds a variable plural embodiment $/ F /$, which is manifested at $w$ by exactly the members $m m$ of $F(w)$ and constituted at $w$ by $\mathrm{mm} / F$. This is a rigid plural embodiment, whose modal profile $f$ is such that for each world-time $u$ other than $w$ :

$$
f(u)= \begin{cases}F(w) & \text { if } F(w)=F(u) \\ \varnothing & \text { otherwise. }\end{cases}
$$

That is $m m / F$ exist at a world-time $u$ if the objects $m m$ that $F$ maps to $w$ exist and verify $F$ at $u$. Consider the queue at the box office again. For simplicity, suppose that two individuals, $a$ and $b$, queue at a given world-time $w_{1}$ and two more $b$ and $c$ queue at $w_{2}$ and $w_{3}$. No individuals, however, queue at the box office at any other world-time. In this case, the principle of generation $F$ is such that $F\left(w_{1}\right)=\{a, b\}$, $F\left(w_{2}\right)=\{b, c\}, F\left(w_{3}\right)=\{b, c\}$ and $F(u)=\varnothing$ for each other world-
${ }^{51}$ Notice that two principles of generation can still differ even if they are satisfied by the same bare plurality at each and every time within a given world. 
time $u$. Now, / $F /$ is constituted by the rigid embodiment $a, b / F$ at $w_{1}$ and $b, c / F$ at $w_{2}$ and $w_{3}$.

The variable existence and identity postulates come down to this:

Every variable modal profile is exemplified by a unique variable plural embodiment.

\subsection{A Formal Framework}

We can extend the formal framework to accommodate variable plural embodiments. To each rigid plural embodiment there corresponds a variable plural embodiment with the same modal profile. So, we could in principle conceive of a rigid embodiment as a special case of a variable embodiment, one whose principle of generation is satisfied by the same bare plurality at every world at which it is satisfied. This invites the question again whether to add yet another sort of variable for variable plural embodiments or simply expand the range of the newly added variables to range over variable plural embodiments. Similar considerations as above suggest we add a new style of variable for variable plural embodiments in the interest of perspicuity. ${ }^{52}$

We introduce another style of variable, $\overline{\overline{x x}}, \overline{\overline{y y}}, \overline{\overline{z z}}, \ldots$, to range over variable plural embodiments. Next we introduce a binary relational expression, $\overline{x x} \triangleleft \overline{\overline{x x}}$, which is implicitly relativized to a world-time and read: " $\overline{x x}$ constitutes $\overline{\overline{x x}}$ ".

This temporary - and contingent - constitution relation is governed by a uniqueness condition:

$$
\overline{x x} \triangleleft \overline{\overline{x x}} \rightarrow \forall \overline{y y}(\overline{y y} \triangleleft \overline{\overline{x x}} \rightarrow \overline{x x}==\overline{y y})
$$

This says that at most one rigid plural embodiment constitutes a given variable plural embodiment at a given world-time.

We can define a "one of" predicate for the new style of variable in terms of $\prec$ as governed by the principle:

$$
x \prec_{V} \overline{\overline{x x}} \leftrightarrow \exists \overline{x x}(\overline{x x} \triangleleft \overline{\overline{x x}} \wedge x \prec \overline{x x}) \quad\left(\prec_{V}\right)
$$

Variable plural embodiments are not extensional: at a given worldtime, the queue at the box office may share all of its members with the search committee and nevertheless remain different from it.

We define an existence predicate $E$ for the new style of variable in terms of constitution. For a variable plural embodiment to exist at a world-time is for some bare plurality to manifest the functional condition associated with it:

$$
E \overline{\overline{x x}} \leftrightarrow \exists \overline{x x}(E \overline{x x} \wedge \overline{x x} \triangleleft \overline{\overline{x x}})
$$

Variable embodiments are not rigid. Alice may be one of the members of the committee at a given time, she is not necessarily one of

\begin{abstract}
${ }^{2}$ Strictly speaking, we could, if we wanted, recast the entire framework with a single sort of variable for variable plural embodiments as rigid plural embodiments are special case of them, bare pluralities are a special case of rigid plural embodiments, and individuals are a special case of bare pluralities.
\end{abstract}


them. She would have failed to be one of them at the given time had she declined the invitation to serve. And even if Beth is not one of members of the committee at a given time, she might have been one had she joined the committee earlier.

At this stage, we are in a position to offer a schematic regimentation of the variable plural existence postulate. First, let us introduce $\operatorname{Func}(A)$ as an abbreviation for the complex formula:

$$
\square \forall x x(A(x x) \rightarrow \forall y y(A(y y) \leftrightarrow y y=x x))
$$

This tells us that $A$ is a plural condition satisfied by at most one bare plurality at each world-time. We may now regiment a variable existence postulate as follows:

$$
\begin{gathered}
\operatorname{Func}(A) \rightarrow \square \forall x x(A(x x) \wedge E x x \rightarrow \\
\exists \overline{\overline{x x}} \square \forall \overline{x x}(\overline{x x} \triangleleft \overline{\overline{x x}} \leftrightarrow \overline{x x}==x x / A))
\end{gathered}
$$

Whenever a functional plural condition $A$ is exemplified by a bare plurality that exists, there is a variable plural embodiment that is necessarily constituted by the rigid plural embodiment, if there is one, that consists of the individuals that satisfy $A$ as they exemplify the condition. 53

Two variable plural embodiments are the same only if they determine the same principle of generation. But if we continue to identify necessarily equivalent conditions, we may simply identify variable plural embodiments with the same constitution conditions:

$$
\overline{\overline{x x}}==\overline{\overline{y y}} \leftrightarrow \square \forall x x(\overline{x x} \triangleleft \overline{\overline{x x}} \leftrightarrow \overline{y y} \triangleleft \overline{\overline{y y}})
$$

It is a simple matter to modify the models of the theory of rigid plural embodiments to accommodate the existence and identity postulates: variable plural embodiment variables are taken to range over variable modal profiles. So, consistency again is not an issue with the framework.

\subsection{Variable Plural Embodiments and their Parts}

There are at least two ways in which something may be a part of a variable plural embodiment. A rigid plural embodiment may be a part of the variable embodiment at a certain world-time, for example, the search committee may include the members of the senior search committee at one world-time even if they are not part of the committee at another time. But a variable plural committee may be part of another variable plural committee in a way that is not relative to a world-time: the senior search committee is part of the senior committee, but there is no reason to think of this relation as temporary.

\footnotetext{
${ }^{53}$ Recall the definition of $x x / A$ as the rigid plural embodiment consisting of $x x$ as they exemplify $A$.
} 
To accommodate the first case, we will take a variable plural embodiment $/ F /$ to have as parts at a given world-time $w$ whatever rigid plural embodiments stand in the timeless relation $\leq_{R}$ to the rigid plural embodiment $\mathrm{mm} / \mathrm{F}$ that constitutes $/ F /$ at $w$ :

First Variable Plural Parthood Postulate: If / F/ is constituted by a rigid plural embodiment $m m / F$ at a world-time $w$, then all parts of $\mathrm{mm} / \mathrm{F}$ are themselves parts of $/ F /$ at $w$.

By way of example, compare the queue at the box office, itself a variable plural embodiment, with the rigid plural embodiments below:

- Alice, Beth, and Charles as they queue at the box office

- Alice, Beth, and Charles as they stand close to each other

- Alice, Beth and Charles as they are distinct from each other

If at a given world-time $w$ the queue at the box office consists of the rigid plural embodiment of Alice, Beth, and Charles as they queue at the box office, then every parts of the rigid plural embodiment becomes at $w$, part of the queue. In particular, each of the other two rigid plural embodiments count as parts of the queue at $w$.

More formally:

$$
\overline{y y} \leq_{R}^{\prime} \overline{\overline{x x}} \leftrightarrow \exists \overline{x x}\left(\overline{x x} \triangleleft \overline{\overline{x x}} \wedge \overline{y y} \leq_{R} \overline{x x}\right) \quad\left(\leq_{R}^{\prime}\right)
$$

We now look at the second type of case. Sometimes a variable plural embodiment is part of another in a way that is not to be relativized to a world-time. Even if the senior search committee shares exactly the same members as the queue at the box office, we want to make sense of the fact that only one of them can be said to be part of the search committee. The queue is never a part of the search committee - even if the bare plurality of its members may at a given world-time be part of the search committee.

One simple suggestion at this point is to take one to be part of another if and only if necessarily, whatever constitutes one is part of whatever constitutes the other.

Second Variable Plural Parthood Postulate: A variable plural embodiment / F/ is part of a variable plural embodiment / G/ if and only if for each and every world-time $w$, the rigid plural embodiment $\mathrm{mm} / \mathrm{F}$ that constitutes $/ F /$ at $w$ is part of the rigid plural embodiment $n n / G$ that constitutes /G/ at $w$.

The search committee may consist of two subcommittees devoted to different searches. Each search committee is part of the larger committee because necessarily, whatever rigid plural embodiment 
constitutes the former is part of whatever rigid plural embodiment constitutes the latter. So, in particular, being a subcommittee of a committee is not merely a matter of having some of its members as members. In symbols:

$$
\overline{\overline{x x}} \leq_{V} \overline{\overline{y y}} \leftrightarrow \square \forall \overline{x x} \forall \overline{y y}\left(\overline{x x} \triangleleft \overline{\overline{x x}} \wedge \overline{y y} \triangleleft \overline{\overline{y y}} \rightarrow \overline{x x} \leq_{R} \overline{y y}\right) \quad\left(\leq_{V}\right)
$$

By (U) above, we know that $\triangleleft$ is guaranteed to be a functional relation: each variable plural embodiment is constituted by at most one bare plurality at a given world-time $w$. This observation plays a role in the derivability of the axioms of core mereology for $\leq_{V}$ :

$$
\begin{aligned}
& \text { Reflexivity: } \overline{\overline{x x}} \leq_{V} \overline{\overline{x x}} \\
& \text { Transitivity: } \overline{\overline{x x}} \leq_{V} \overline{\overline{y y}} \wedge \overline{\overline{y y}} \leq_{V} \overline{\overline{z z}} \rightarrow \overline{\overline{x x}} \leq_{V} \overline{\overline{z z}} \\
& \text { Anti-symmetry: } \overline{\overline{x x}} \leq_{V} \overline{\overline{y y}} \wedge \overline{\overline{y y}} \leq_{V} \overline{\overline{x x}} \rightarrow \overline{\overline{x x}}==\overline{\overline{y y}}
\end{aligned}
$$

Consider reflexivity, first. Since necessarily, $\overline{\overline{x x}}$ is necessarily constituted by one and the same rigid plural embodiment as $\overline{\overline{x x}}$, whatever constitutes one at each given world-time is trivially part of what constitutes the other. Thus $\overline{\overline{x x}}$ is part of $\overline{\overline{x x}}$.

Transitivity is another simple consequence of the functionality of $\triangleright$ and the transitivity of $\leq_{R}$. Given three variable plural embodiments $\overline{\overline{x x}}, \overline{\overline{y y}}$, and $\overline{\overline{z z}}$, If $\overline{\overline{x x}}$ is part of $\overline{\overline{y y}}$, then necessarily, whatever rigid plural embodiment constitutes $\overline{\overline{x x}}$ at a world-time $w$ is part of whatever constitutes $\overline{\overline{y y}}$ at $w$. And if $\overline{\overline{y y}}$ is part of $\overline{\overline{z z}}$, then necessarily, whatever rigid plural embodiment constitutes $\overline{\overline{y y}}$ at a world-time $w$ is part of whatever constitutes $\overline{\overline{z z}}$ at $w$. By transitivity of $\leq_{R}$, whatever rigid plural embodiment constitutes $\overline{\overline{x x}}$ at a world-time $w$ is part of whatever constitutes $\overline{\overline{z z}}$ at $w$.

As for anti-symmetry, suppose that necessarily, whatever rigid plural embodiment constitutes $\overline{\overline{x x}}$ at a world-time $w$ is part of whatever constitutes $\overline{\overline{y y}}$ at $w$, and vice versa. Since, by (U), the rigid plural embodiments that constitute $\overline{\overline{x x}}$ and $\overline{\overline{y y}}$, respectively, are mutual parts of each other, by anti-symmetry for $\leq_{R}$, they are one at the same at each world-time at which the variable plural embodiments exist. So, by ( $V$-Identity), $\overline{\overline{x x}}$ and $\overline{\overline{x x}}$ are one and the same variable plural embodiment.

Unlike rigid plural embodiments, however, variable plural embodiments are governed by strong supplementation:

$$
\text { Strong Supplementation: } \overline{\overline{x x}} \nless_{V} \overline{\overline{y y}} \rightarrow \exists \overline{\overline{z z}}\left(\overline{\overline{z z}} \leq_{V} \overline{\overline{x x}} \wedge \neg \overline{\overline{z z}} \circ_{V} \overline{\overline{y y}}\right)
$$

To justify this claim, suppose that a variable plural embodiment $\overline{\overline{x x}}$ is not part of another $\overline{\overline{y y}}$. Given $\left(\leq_{V}\right)$, it is possible for $\overline{\overline{x x}}$ to be constituted by a rigid plural embodiment $\overline{x x}$ that is not part of whatever 
rigid plural embodiment $\overline{y y}$ constitutes $\overline{\overline{y y}}$ at the relevant world-time. But consider the condition: $\forall x\left(x \prec x x \leftrightarrow\left(x \prec_{V} \overline{\overline{x x}} \wedge x \nprec_{V} \overline{\overline{y y}}\right)\right)$. This is a functional condition satisfied at each given world-time $w$ by the bare plurality $x x$ of members of $\overline{\overline{x x}}$ that are not members of $\overline{\overline{y y}}$. Since the condition is indeed satisfied by some individuals at the world of evaluation, by ( $V$-Existence), there is a variable plural embodiment $\overline{\overline{z z}}$ generated by the condition. It is routine to check that the variable embodiment in question is part of $\overline{\overline{x x}}$ but fails to overlap $\overline{\overline{y y}}$.

Strong supplementation entails a principle of extensionality for variable plural embodiments:

$$
\text { Extensionality: } \forall \overline{\overline{z z}}\left(\overline{\overline{z z}} \leq_{V} \overline{\overline{x x}} \leftrightarrow \overline{\overline{z z}} \leq_{V} \overline{\overline{x x}}\right) \rightarrow \overline{\overline{x x}}==\overline{\overline{y y}}
$$

This tells us that two variable plural embodiments are one and the same if, and only if, they have the same variable plural embodiments as parts. But given the distinction between rigid and variable plural embodiments, this form of extensionality is much weaker than it may seem. This principle is perfectly consistent with the observation that two variable plural embodiments may share exactly the same rigid plural parts and nevertheless remain numerically distinct. 54

In addition to this, it is not unreasonable to assume a principle of unrestricted composition:

$$
\begin{aligned}
& \text { Unrestricted Fusion: } \exists \overline{\overline{x x}} \varphi(\overline{\overline{x x}}) \rightarrow \exists \overline{\overline{x x}}\left(\forall \overline { \overline { y y } } \left(\varphi(\overline{\overline{y y}}) \rightarrow \overline{\overline{y y}} \leq_{V}\right.\right. \\
& \left.\overline{\overline{x x}}) \wedge \forall \overline{\overline{y y}}\left(\overline{\overline{y y}} \leq_{V} \overline{\overline{x x}} \rightarrow \exists \overline{\overline{z z}}\left(\varphi(\overline{\overline{z z}}) \wedge \overline{\overline{y y}} \circ_{V} \overline{\overline{z z}}\right)\right)\right)
\end{aligned}
$$

For suppose some $\overline{\overline{x x}}$ are such that $\varphi(\overline{\overline{x x}})$. And consider the condition a bare plurality $x x$ satisfies when it fuses the bare pluralities that are coextensive with one or another variable plural embodiment satisfying $\varphi$. This is a functional condition since bare pluralities are governed by classical extensional mereology. Now: there is by (Comprehension), a bare plurality that satisfies this condition, which means, by ( $V$-Existence), that there is a variable plural embodiment which is necessarily constituted by rigid plural embodiments consisting of bare pluralities satisfying the functional condition in question.

To summarize, we have characterized, first, $\leq_{R}^{\prime}$ as a temporary and contingent relation a rigid plural embodiment may bear to a variable plural embodiment at a given world-time. Unlike this relation, $\leq_{V}$ is not to be relativized to a world-time and turns out to be governed by the axioms of classical mereology.

\section{Conclusion}

It is time to take stock. We have suggested a hylomorphic conception of groups conceived not as complex material objects over and above their members, but rather as variable plural embodiments constituted
${ }^{54}$ It is not, for example, to be confused with two considerably stronger but false principles:

$$
\text { Extensionality: } \forall \overline{z z}\left(\overline{z z} \leq_{R} \overline{\overline{x x}} \leftrightarrow\right.
$$

$$
\left.\overline{z z} \leq_{R} \overline{\overline{x x}}\right) \rightarrow \overline{\overline{x x}}==\overline{\overline{y y}}
$$

There is no reason to expect this form of extensionality to hold, since two different variable plural embodiments may at a given world-time, share all of their rigid plural parts and nevertheless remain different. Likewise for an even less plausible principle:

$$
\begin{aligned}
& \text { Extensionality: } \forall z z\left(z z \leq_{R} \overline{\overline{x x}} \leftrightarrow\right. \\
& \left.z z \leq_{R} \overline{\overline{x x}}\right) \rightarrow \overline{\overline{x x}}==\overline{\overline{y y}}
\end{aligned}
$$

Two variable plural embodiments may share the same material parts at a given world-time $w$ and nevertheless remain numerically distinct. 
by rigid plural embodiments at a given world-time. Rigid plural embodiments in turn consist of some individuals as they exemplify a certain complex condition. We should now make sure that the identification of groups with variable plural embodiments does justice to the three constraints we outlined at the outset.

We note first that membership to a group - conceived as variable plural embodiment - may be temporary and contingent. This is simply because some individuals may satisfy the functional condition that generates the relevant variable plural embodiment we identify with the group only temporarily and contingently. While Alice, Beth, and Charles may at a given world-time satisfy the principle of generation for a queue at the box office, they need not do so at other world-times. Likewise for a committee. The fact that some individuals satisfy the complex functional condition associated with the variable plural embodiment with which we have identified the committee is perfectly compatible with the fact that other individuals do so at some other world-times.

The identification of groups with variable plural embodiments is similarly tailored to accommodate coincidence. Although the search and graduate committees are generated by two different functional conditions, it should be no surprise that the same individuals can, at a given world-time, satisfy one and the other. The functional conditions remain numerically different because it is possible for them to be satisfied by two different bare pluralities of individuals. So, the very same individuals who meet on Tuesday as the members of the search committee may meet on Thursday as the members of the graduate committee.

The account of what it is for an individual to be a member of a group at a given world-time is for the individual to be one of some individuals satisfying a complex condition, which is itself a principle of generation for the variable plural embodiment with which we have the group. This account illuminates the difference between highly structured groups like committees and less cohesive groups like queues. The main difference is to be located in the principles of generation corresponding to each and whether or not they are a source of shared intentionality and other complex features of committees.

Last, but not least, we have outlined an account of what it is for a group to be part of another. More generally, we have distinguished at least two different ways in which something may be part of a group. One of them is to be relativized to a world-time as it has to do with what is part of whatever constitutes the variable embodiment at a world-time. The other, however, requires no such relativization: one group is part of another if and only if for each and every worldtime, whatever rigid plural embodiment constitutes one is part of 
whatever rigid plural embodiment constitutes the second. While the admissions committee may be coextensive with the senior search committee, which is itself part of the search committee, the admissions committee is itself not part of the search committee. The reason is simple: the admissions committee may at given world-time $w$ be constituted by a rigid plural embodiment that is not itself part of whatever rigid plural embodiment constitutes the search committee at $w$.

The formal regimentation of the framework is an extension of modal quantified plural logic for which it is simple to find models, which means that unlike traditional formulations of hylomorphism, the present account is perfectly consistent. The mere consistency of the proposal is, by itself, not a reason to adopt it. However, we have suggested that the concept of a plural embodiment should be attractive to proponents of hylomorphic composition, and once it becomes available, it enables us to provide an account of groups that appears to do better than extant accounts when it comes to the constraints we outlined at the outset and the further judgment that at a given world-time, a group is nothing over and above the individual members as they satisfy a certain condition.

\section{References}

P. Bricker. Quantified Modal Logic and the Plural De Re. Midwest Studies in Philosophy, 14(1):372-394, 1989.

D. Copp. What collectives are: Agency, individualism and legal theory. Dialogue, 23(2):249-269, 1984.

M. J. Cresswell and G. E. Hughes. A New Introduction to Modal Logic. Routledge, 2012.

N. Effingham. The Metaphysics of Groups. Philosophical Studies, 149

(2):251-267, 2010.

B. Epstein. The Ant Trap: Rebuilding the Foundations of the Social Sciences. Oxford, 2015.

M. Fairchild. A Paradox of Matter and Form. Thought, 5, 2017.

K. Fine. Acts, Events and Things. In Sixth International Wittgenstein Symposium, Kirchberg-Wechsel (Austria), pages 97-105, 1982.

K. Fine. Things and their Parts. Midwest Studies in Philosophy, 23(1): 61-74, 1999 .

K. Fine. Response to Kathrin Koslicki. dialectica, 61(1):161-166, Mar. 2007. 
S. Florio. Untyped Pluralism. Mind, 123(490):317-337, Apr. 2014.

P. Fritz and J. Goodman. Counting incompossibles. Mind, page fzwo26, 2017. DoI: 10.1093/mind/fzwo26. URL +http://dx. doi. org/10.1093/mind/fzw026.

J. Goodman. Matter and Mereology. Forthcoming in Noûs, 2016.

K. Hawley. Social ontology. Journal of the American Philosophical Association, 2017.

A. P. Hazen. Against Pluralism. Australasian Journal of Philosophy, 71 (2):132-144, 1993.

D. Z. Korman. Strange Kinds, Familiar Kinds, and the Charge of Arbitrariness. Oxford Studies in Metaphysics, 5(119-144), 2010.

K. Koslicki. The Structure of Objects. Oxford University Press, 2008.

D. Lewis. Noneism or Allism? Mind, 99(393):23-31, 1990. ISSN o0264423, 14602113. URL http://www. jstor.org/stable/2254888.

Ø. Linnebo. Plural quantification. In E. N. Zalta, editor, The Stanford Encyclopedia of Philosophy. Metaphysics Research Lab, Stanford University, summer 2017 edition, 2017.

Ø. Linnebo and D. Nicolas. Superplurals in english. Analysis, 68(3): 186-197, 2008.

Ø. Linnebo and A. Rayo. Hierarchies: Ontological and Ideological. Mind, 121(482):269-308, 2012.

G. Macdonald and P. Pettit. Semantics and Social Science. Routledge \& Kegan Paul, 1981.

T. McKay. Plural Predication. Oxford scholarship online. Clarendon Press, 2006.

T. Merricks. Objects and Persons. Oxford University Press, 2001.

G. Priest. Towards Non-Being: The Logic and Metaphysics of Intentionality. Oxford University Press, 2005.

A. Quinton. Social Objects. In Proceedings of the Aristotelian society, volume 76 , pages $1-27$. JSTOR, 1975.

A. Rayo. Absolute Generality, chapter Beyond Plurals, pages 220-254. Oxford University Press, 2006.

K. Ritchie. What Are Groups? Philosophical Studies, 166(2):257-272, 2013. 
D.-H. Ruben. Social Wholes and Parts. Mind, 92(366):219-238, 1983.

D.-H. Ruben. The Metaphysics of the Social World. Routledge \& Kegan Paul, 1985 .

T. Sider. Four Dimensionalism: An Ontology of Persistence and Time. Oxford University Press, 2001.

P. M. Simons. Parts: A Study in Ontology. Oxford University Press, 1987.

G. Uzquiano. The Supreme Court and the Supreme Court Justices: A Metaphysical Puzzle. Noûs, 38(1):135-153, 2004.

P. van Inwagen. Material Beings. Cornell University Press, 1990.

A. Varzi. Mereology. In E. N. Zalta, editor, The Stanford Encyclopedia of Philosophy. Metaphysics Research Lab, Stanford University, winter 2016 edition, 2016. 